\title{
Infrastructure Operator Interfaces for Passenger Evacuation
}

\author{
Harald Widlroither ${ }^{1}$ Nicola Reggiani ${ }^{2}$ Elisa Landini ${ }^{2}$ Jan-Paul Leuteritz $^{3}$ \\ ${ }^{1}$ Fraunhofer IAO, Germany \\ ${ }^{2}$ Università di Modena e Reggio Emilia, Italy \\ ${ }^{3}$ Universität Stuttgart IAT, Germany
}

\begin{abstract}
Developing proof-of-concept solutions for new integrated rescue support systems is challenging, due to the different restrictions and procedures at different installation sites. One way of dealing with this conflict is the provision of one prototype for a mininum- and one for the maximum number of functions. This article presents a support module for the operators of traffic infrastructures, developed with minimum functionalities, which allows for implementing an automated critical event detection and passenger rescue, supported by the SAVE ME system. Here, its functions, the implementation and test results from two pilot tests are described.
\end{abstract}

Keywords: user interface, evacuation, operator, traffic infrastructure, emergency

\section{The SAVE ME System}

Closed infrastructures, such as road tunnels and metro stations can transform into fatal traps for travelers when fires or other dangerous events occur. Travelers need to rescue themselves, as waiting for rescue teams might take too long [1]. A substantial number of travelers cannot be expected to behave correctly on instinct [2]. Some passengers, for example, tend to stay in their vehicles when they are caught in a fire event in a road tunnel thus facing smoke intoxication and death.
Thus, a clear warning and an instruction on how to escape and where to escape to is of vital importance.

The SAVE ME project [3], co-founded by the European Commission in the $7^{\text {th }}$ Framework Programme, has created a technical system for supporting mitigation and passenger rescue in traffic infrastructure, such as tunnels, or metro stations.

An integrated sensor system detects emergencies such as fire, smoke, or other dangerous situations. As a consequence, the system detects the location of the dangerous event (e.g., the fire), andthe positions of passengers who have installed the SAVE ME app on their mobile devices. The SAVE ME Decision Support System (DSS) then calculates the optimal evacuation routes for all these passengers. The travellers equipped with a SAVE ME enabled mobile device receive the necessary, personalized guidance information in form of a map on their mobile device. The route calculation takes the mobility characteristics of the passengers into account: travellers in wheelchairs, for example, are provided with a route that avoids staircases or other critical obstacles. For passengers without SAVE ME enabled mobile devices, selfrescue information is shown on displays installed at the walls throughout the infrastructure [3].

Rescue teams equipped with a SAVE ME enabled handheld device can then localize the positions of possibly trapped 
passengers and are guided to their location.

\section{The SAVE ME OpS-UI}

The operator of the infrastructure thus plays a key role in the evacuation process. Together with the passengers, who can use the SAVE ME app on their mobile phones and the rescue teams, who are also provided with a handheld device, they are one of the three groups of users that interact with the SAVE ME system by using a user interface.

\subsection{User- \& technical Requirements}

In the development of the SAVE ME system, the characteristics of two types of infrastructure operators were taken into account at the development stage:

Policemen who monitor the Gotthard Strassentunnel in Göschenen, Switzerland.

Passenger Service Operators of the Newcastle metro in Great Britain.

The job of an operator is characterized by high time pressure and great responsibility. Most of them work either alone or in a team of two. In uncritical situations, they mostly monitor the correct functioning of the traffic infrastructure using a lot of information systems at a time, including several computer monitors, video monitors and other sources of information. In emergency situations, they follow a clearly defined procedure. Workload increases enormously as they need to communicate with a lot of different persons in a very short period of time, while continously monitoring the infrastructure. Under these circumstances, it is clear that the SAVE ME system must

- Request only a minimum number of interactions from the operators

- Provide means to perform these interactions in a minimum time
- Provide a highly intuitive user interface that does hardly require any training.

In addition to these factors it seemed appropriate to provide a user interface that was easy to carry, so it would stay with the operator even if he moves across the room. It should also have an independent power supply for the case power cuts during an emergency.

Independent from the type of test site - road tunnel or metro station, the operator has to perform the following tasks in order to ensure that the evacuation supported by SAVE ME will run efficiently and effectively:

- Perceive the alert, either by using the OpS-UI, or by using other sources of information, such as calls from SOS telephones, or video surveillance and determine location, severity and other key factors of the alert condition.

- Check if the alert is correct and, if yes, inform authorities or colleagues according to the site-specific emergency protocol.

- Check the evacuation routes provided by SAVE ME (which is also due to internal regulations at different places where SAVE ME has been tested: A human operator always needs to take the final decision of evacuation). If the infrastructure has additional means installed to support passenger egress, such as a P.A. or radio broadcast system, then the operator will now use it.

- Monitor the egress of the passengers.

- Keep contact with rescue teams and other involved authorities to aid in the rescue activities.

\subsection{Implementation}

In order to enable the operator to perform all these tasks most efficiently, SAVE 
ME developed a User Interface for the Operator Module: the OpS-UI (Operator Support - User Interface).

Proving a concept for a technology such as the OpS-UI as a part of a new evacuation system is difficult. At our two pilot sites, there were substantial differences concerning the type of operators and their background and rights, evacuation procedures and other factors. In order to show that it was possible to implement all functions envisioned in SAVE ME, even if they only applied to specific contexts (such as an automated function to inform defined third parties about a detected event), a web portal was designed, based on HTML. However, it was also intended to use the pilot prototype of the OpS-UI in order to convince operators and stakeholders at the pilot sites of the benefits of SAVE ME. It was to be demonstrated that SAVE ME core functions works with a minimum of restrictions, thus being most easily applied in any infrastructure and context. Therefore, another instance of the OpS-UI was developed for the pilot tests, with a more limited range of functionality. In this article, we present the latter: The instance of the OpS-UI that only holds these main functionalities and that was put to test at the SAVE ME pilot sites.

For the purpose of pilot testing and proof-of-concept, the OpS-UI was implemented on the iPad® 3.After the successful login, the user can choose between four different screens, using the tab-navigation at the bottom:

- Map, which provides two types of map, to be switched between using two buttons. One map shows the escape routes planned by the system, the other shows the current positions of all actors and the positions of the dangerous events. On the map, the operator can use on-off switches to close or open single paths in the in- frastructure, thus influencing the flow of passengers. This is necessary for the case that a path is blocked, without the SAVE ME sensors detecting, it as a consequence of a critical event.

- Messages, which displays logs of all system events starting with the operator's login.

- Travelers, which provides an option to send text messages to the mobile devices of the travelers, and

- Rescue Teams, which offers an option to send text messages to the mobile devices of the rescue team leaders.

On the right side of the screen there is a frame that is always visible, no matter which tab is currently open. It provides a list of the steps the operator is supposed to be taking, and which can be adapted to reflect any emergency procedure at any infrastructure. It also includes a button to request a new route calculation.

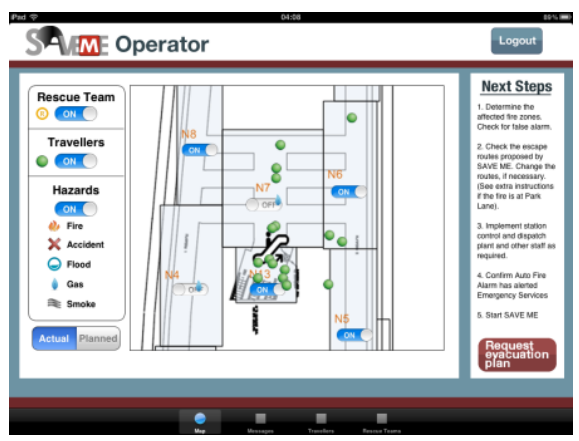

Fig. 1: Screenshot of the Ops-UI, map-tab (iPad®-version).

The iPad®-application, which represents the graphical user interface, is connected to the other SAVE ME components via a java server. The java server itself is connected to the SAVE ME Decision Support System (DSS), which serves as a hub for exchanging information between all connected modules. When there is an ac- 
tive session, the relevant information is exchanged in XML format between the DSS and the java server, while the app accesses the information through HTTP GET requests to the java server.

Thanks to the modular OPS's clientserver structure, the java server can manage as many iPads as necessary according to the topology of the environment.

\section{Technical evaluation of the OpS-UI}

The entire SAVE ME system was tested at two pilot sites in June 2012: at the Colle Capretto Road Tunnel in Italy, an accident involving multiple cars was simulated in order to compare the effectiveness of egress with and without the SAVE ME system. A total of 32 participants from different age groups, equally divided into an experimental and a control group participated in the trial.

At the Monument Station of the metro network in Newcastle upon Tyne, UK, two runs of different evacuation scenarios were performed with a group of 21 participants. The participants were dispersed across the two platforms, according to a pre-defined pattern. This served to avoid a test situation in which too many participants would be found in the same spot and thus would share the same rescue path. Some participants took the role of mobility impaired users by adding the respective information in their user profile.
Users with real mobility issues did not participate in the test, due to security reasons.

In this pilot test, 10 iPads have been installed and their reliability and accuracy of the Operator Module were measured. The Operator Module never disconnected from the DSS during the entire time of the test, which means that it always displayed the real-time data provided by the other modules. These variables were also found to meet the quality criteria defined ex ante for the evaluation of the system. Thus, the OpS-UI was evaluated successfully and is thus ready for deployment.

\section{References}

[1] Schweizerische Eidgenossenschaft (2011). Sicherheit im Tunnel - "Security in the tunnel" GotthardStrassentunnel - Official homepage http://www.afbn.ch/Sicherheit-imTunnel.106.0.html, last access on February 21st, 2011.

[2] Färber B. \& Färber B. (2010) Verhaltensanweisungen bei Notsituationen in Straßentunneln. Berichte der Bundesanstalt für Straßenwesen (BASt), M 212. Bergisch Gladbach: Wissenschaftsverlag NW.

[3] SAVE ME Description of Work. EC Grant Agreement 234027. FP7-SST2008-RTD-1 